\title{
The effect of question order on responses to multiple-choice questions
}

\author{
Kara Gray, Sanjay Rebello and Dean Zollman \\ Physics Department, Kansas State University, Manhattan, KS 66506
}

\begin{abstract}
Educators and researchers often make the assumption that the order of test or survey questions is unimportant. Is this assumption valid? This study investigates how the order of two related FCI questions (\#13 and 14) affects students' responses. This study also investigates the effect an unrelated FCI question (\#23) has on answers to the above problems. Four versions of a survey were administered before and after instruction to 243 students taking an algebra-based physics class. Versions 1 and 2 of the survey included the related physics questions in opposite order. Versions 3 and 4 included the unrelated physics question and one of the above questions. Student responses for the four versions were compared for both the pre- and post-instruction surveys.
\end{abstract}

\section{Introduction}

For years physics education researchers have used surveys and other investigative tools to learn what physics students know and how they think. These researchers often assume that the order of questions does not affect student responses. For as long as teachers have been giving tests, students have been looking for strategies and "tricks" to improve their performance on exams. These methods include "test taking strategies" often taught in high school and some college orientation classes. A common strategy includes looking for the answer or helpful hints in other problems when answering an unfamiliar question. Thus, students' performance may be influenced by their test taking skills or ability to "learn" the physics as they take the test. There is no reason to believe that students forget this skill when they are taking physics education research surveys as opposed to graded exams. This is a serious problem for researchers who are attempting to understand how students think about a series of problems or situations.

Educational researchers have for years studied the effect of question order, beginning with Mollenkopf [1] and others in the 1950s. Most early studies [2] focused on changing the order of the ease of the questions: "easy to hard" and "hard to easy." With the advent of computerized adaptive testing educational researchers have begun to look more closely at the effect of changing the context and order in which test items appear.[3]

The purpose of this paper is to test the aforementioned assumption that the order of questions in a test does not affect student responses to individual questions on the test. The results of our study described here suggest that the order of questions does have an effect on students' responses to multiplechoice questions, including the frequency of correct responses and responses based on common misconceptions. This paper is intended to draw attention to a basic assumption of most research and to initiate a discussion on the validity of this assumption.

\section{Methodology}

We surveyed 243 students enrolled in an algebrabased physics course taught using the lecture/recitation/lab format. Most students taking the class were life science, pre-vet, agriculture or education majors. Approximately $47 \%$ of the students were female and about $70 \%$ had already taken a physics class at the high school or college level. We administered the pre-test a week into the semester and the post-test a week after the students took the second exam, which covered forces. The surveys were administered during lab.

The surveys included three questions from the Force Concept Inventory (FCI).[4] These three questions included two mutually related questions pertaining to Newton's III Law and one unrelated question pertaining to projectile motion. We limited our survey instrument to three questions mainly to limit the number of experimental variables in our study. We used a modified set of distracters developed from students' answers to open-ended versions of the questions. [5]

The first question $(\alpha)$ in Figure 1 is FCI question \#13. It will be referred to as the acceleration scenario. The second question ( $\beta$ ) in Figure 2 is FCI question $\# 14$ and it will be referred to as the non-accelerating scenario. For both questions the correct answer is choice A. The final question is FCI question \#23 in Figure 3, which is referred to as the control question. 
The correct answer to the control question is choice 5 . Questions $\alpha$ and $\beta$ are both related to Newton's III Law. The control question is a projectile motion problem and therefore unrelated.

A large truck breaks down out on the road and receives a push back into town by a small compact car as shown in the figure below.

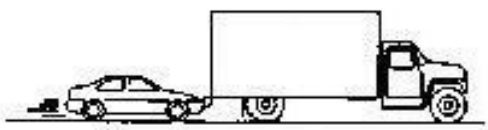

While the car, still pushing the truck, is speeding up to get up to cruising speed:

(A) The force with which the car pushes on the truck is equal to that which the truck pushes back on the car.

(B) The force with which the car pushes on the truck is smaller than that which the truck pushes back on the car.

(C) The force with which the car pushes on the truck is greater than that which the truck pushes back on the car.

(D) The car's engine is running so the car pushes against the truck, but the truck's engine is not running, so the truck does not push against the car.

(E) Neither the car nor the truck exerts any force on each other.

Figure 1: Question $\alpha$; the accelerating scenario.

A large truck breaks down out on the road and receives a push back into town by a small compact car as shown in the figure below.

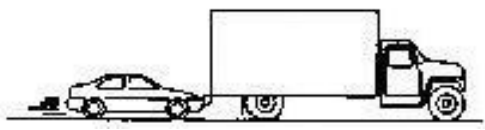

After the car reaches a constant cruising speed at which the driver wishes to push the truck:

(A) The force with which the car pushes on the truck is equal to that which the truck pushes back on the car.

(B) The force with which the car pushes on the truck is smaller than that which the truck pushes back on the car.

(C) The force with which the car pushes on the truck is greater than that which the truck pushes back on the car.

(D) The car's engine is running so the car pushes against the truck, but the truck's engine is not running, so the truck does not push against the car.

(E) Neither the car nor the truck exerts any force on each other.

Figure 2: Question $\beta$; the non-accelerating scenario.

The purpose of this research was to study the effect the order of two related questions ( $\alpha$ and $\beta$ ) would have on student responses and to investigate the effects of the unrelated control question on student responses to questions $\alpha$ and $\beta$. Therefore, four versions of the survey were created with each survey containing two of the three questions.

A bowling ball accidentally falls out of the cargo bay of an airliner as it flies along in a horizontal direction.

As observed by a person standing on the ground and viewing the plane as in the figure below, which path would the bowling ball most closely follow after leaving the airplane?

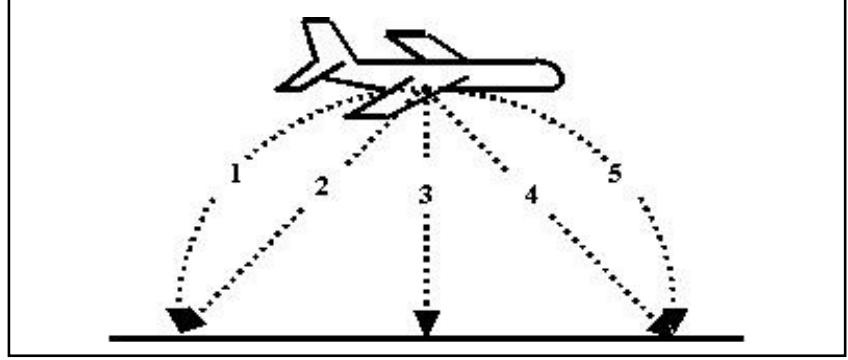

Figure 3: The control question.

Table 1 shows the arrangement of the survey questions and the number of respondents for each version of the survey. Versions 1 and 2 were created to study the effects of the ordering of questions $\alpha$ and $\beta$, while Versions 3 and 4 investigated the effects of the control question. Students were given only one question at a time on a separate sheet of paper that was collected from the students before the second question was handed out. This process ensured that students could not change their answer to question 1 after reading question 2 or answer the questions out of order. T-tests on the scores on the second exam showed no statistical difference in physics ability between the version groups.

\begin{tabular}{|l|c|l|l|}
\hline & $\mathrm{n}$ & Question 1 & Question 2 \\
\hline Version 1 & 64 & Question $\alpha$ & Question $\beta$ \\
\hline Version 2 & 59 & Question $\beta$ & Question $\alpha$ \\
\hline Version 3 & 59 & Control Question & Question $\alpha$ \\
\hline Version 4 & 61 & Control Question & Question $\beta$ \\
\hline
\end{tabular}

Table 1: The order of survey questions.

\section{Results and Discussion}

The frequency of each response for the questions was analyzed using the standard z-tests on the proportion [6] of students who selected that response. The following graphs show the effect of the order and the control question.

Pre-Test

Graphs 1-3 show the frequency of each response (A through $\mathrm{E}$ on Questions $\alpha$ and $\beta$ or 1 through 5 on 
the Control Question) for each question and version of the pre-test.

The placement of question $\alpha$ appeared to have no effect on how students answered this question. While the graph shows minor fluctuations for the different versions, these differences were not statistically significant. It should be noted that no students selected choice $\mathrm{E}$ for question $\alpha$. This observation may mean that students recognized that in an acceleration scenario, the car and truck exert some type of force on each other.

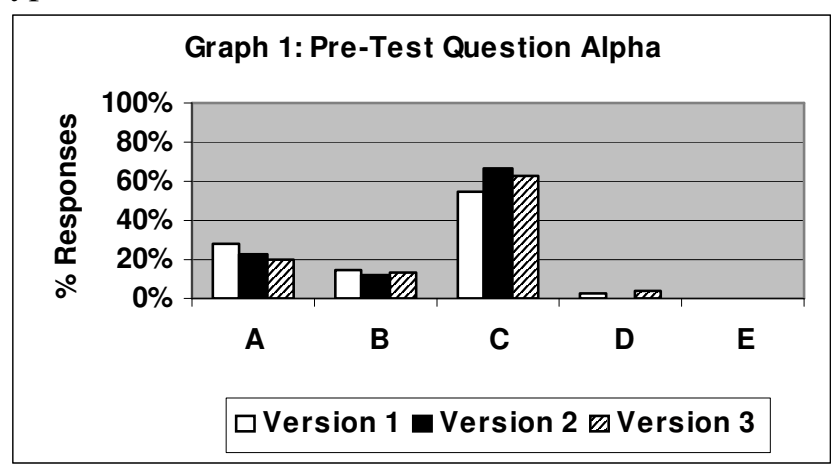

The results for question $\beta$ tell a much more interesting story. There is a statistically significant difference $(\mathrm{z}=2.57, \mathrm{p} \leq 0.0052)$ in the percentage of students who select choice E for version $2(0 \%)$ and those who select choice $\mathrm{E}$ for version $1(9.4 \%)$.

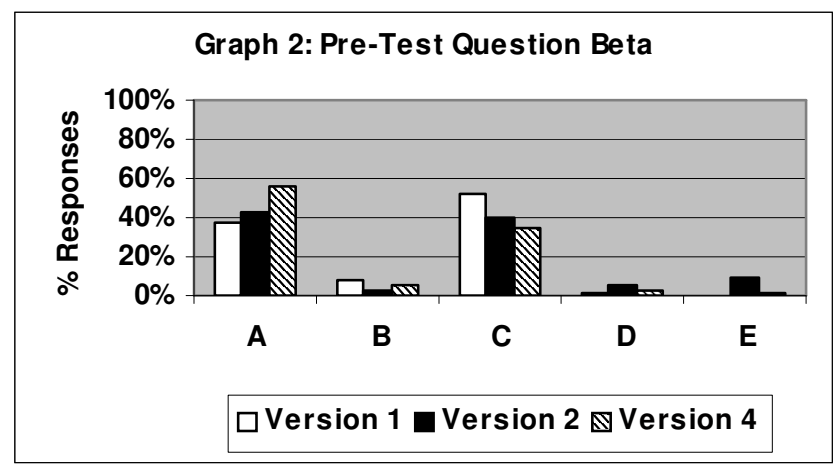

This suggests that students are only likely to think that there are no forces present between the two objects in a non-accelerating situation after they have dealt with the accelerating situation. Students were more likely $(\mathrm{z}=2.06, \mathrm{p} \leq 0.0197)$ to choose the correct answer (A) after answering the control question than when they answered question $\beta$ first (version 2). They were also less likely $(\mathrm{z}=2.03, \mathrm{p} \leq 0.0212)$ to choose answer $\mathrm{C}$, the most common distracter, after answering the control question than when they answered question $\beta$ first. There was also a statistically significant difference $(\mathrm{z}=2.22, \mathrm{p} \leq 0.0132)$ between Versions 3 and 4 , in frequency of response 1 to the control question.

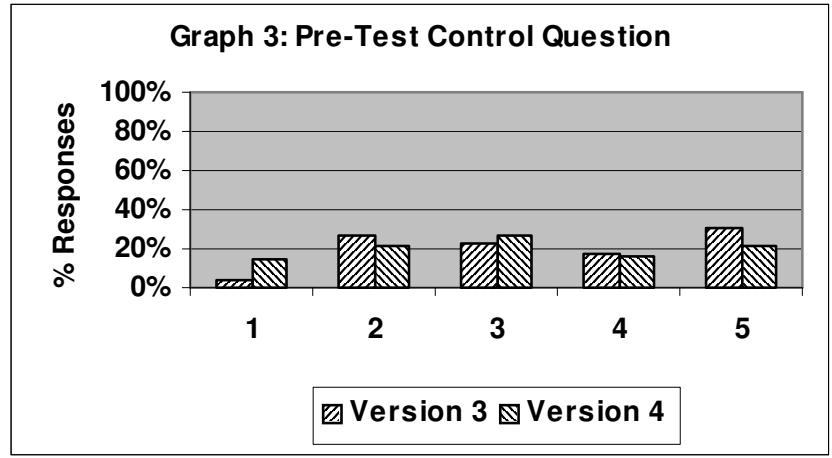

Another notable result from the pre-test is that Post-Test

The results for the post-test surveys are shown in graphs 4-6. The frequency of correct responses improved for the post-test. Yet, choice $\mathrm{C}$ was still the most common selection for question $\alpha$, indicating that students feel the car is exerting a greater force than the truck when they are accelerating.
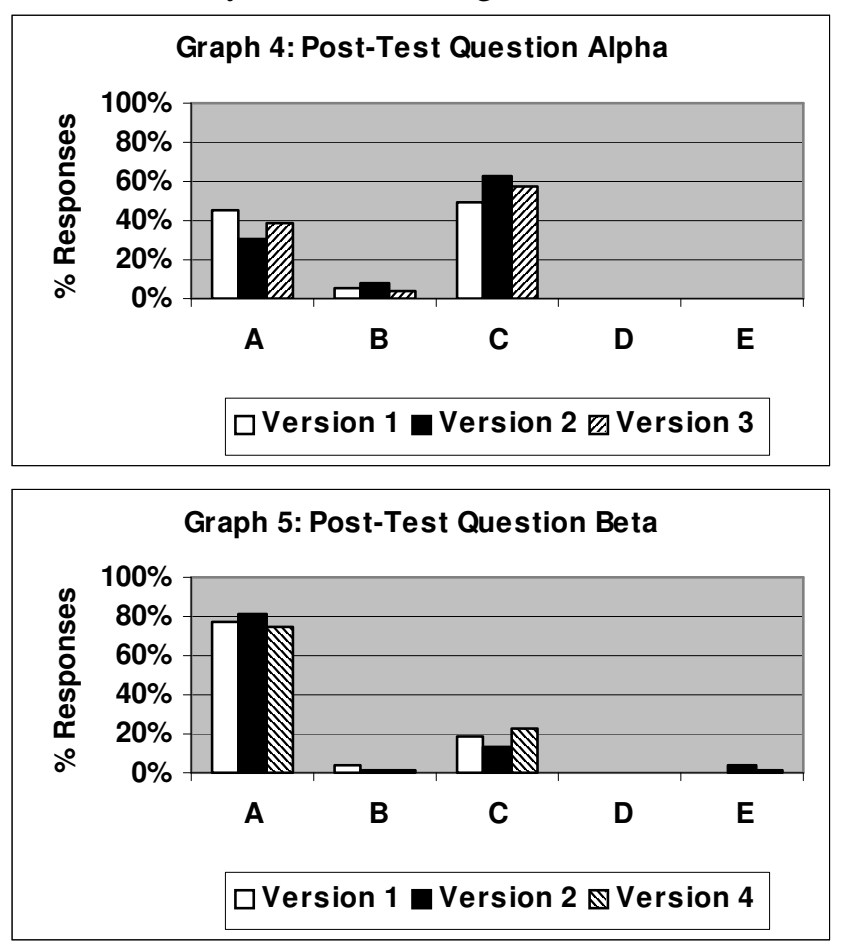

For question $\beta$, the most common selection was choice A, the correct answer. Seventy percent to $80 \%$ of students correctly answered this problem, depending on the version. There were no significant differences based on question order or inclusion of the control question for the post-test. Based on the above result, we speculate that after instruction, student responses are not as likely to be influenced by the question order than before instruction. 


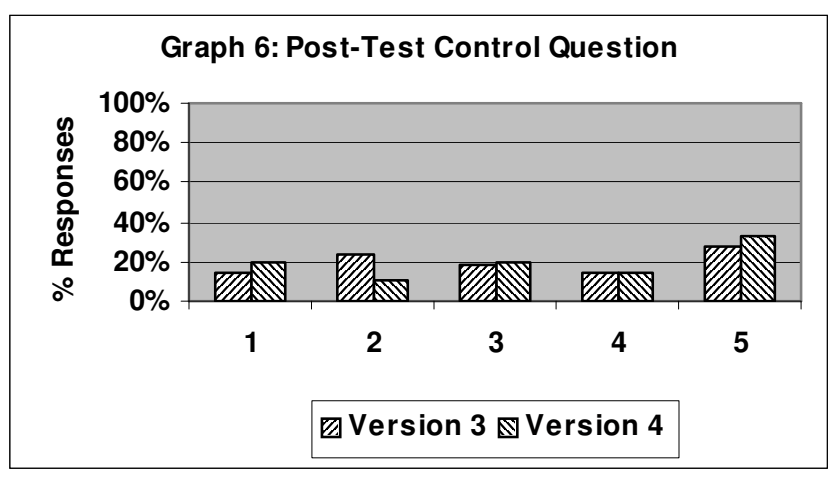

\section{Conclusions}

Our research shows that the order of questions and the inclusion of an unrelated question can have a statistically significant effect on student responses. We used two Newton's III Law questions in our survey, one in which the objects were accelerating and another in which they were moving at a constant speed.

On the pre-test when the accelerating scenario (question $\alpha$ ) was presented first, followed by the nonaccelerating scenario (question $\beta$ ), about $9.4 \%$ of the students responded that no force was present when the objects (car and truck) traveled at a constant speed even though they recognized that a force was present between the accelerating objects. By contrast, when the order of questions was reversed - i.e. the nonaccelerating scenario (question $\beta$ ) was presented first, followed by the accelerating scenario (question $\alpha$ ) there were no responses that stated the non-existence of interaction forces in the non-accelerating case. This difference (i.e. $9.4 \%$ vs. $0 \%$ ) is statistically significant $(\mathrm{z}=2.57, \mathrm{p} \leq 0.0052)$. Thus, it appears that a particular order of questions is more likely to trigger a certain misconception than another order of questions.

It also appears that some students were influenced by the control question. These students fared better on the non-accelerating scenario after being asked an unrelated physics question. Not only was the frequency of correct answers improved, but also the frequency of the most common distracter was decreased. The reason for this is not entirely understood. One possible explanation for this phenomenon is that the unrelated question put students in a "physics mindset." The control problem appears to have encouraged students to begin thinking about the questions as a physicist would.

The results of our study are statistically significant, albeit marginally. Therefore, in our ongoing efforts we will retest our results with another group of students enrolled in a different semester. Based on our present results however, we can state that the assumption that the order of questions is irrelevant should no longer be accepted as simple fact. Instead there is reason to believe that the effect of question order on student response merits further careful investigation and should be considered in the design of survey instruments.

\section{Acknowledgements}

This work is supported in part by NSF grant \# REC0087788 .

\section{References}

1. Mollenkopf, W. G. (1950) "An experimental study of the effects on item analysis data of changing item placement and test-time limit," Psychometrika, 15, 291-315.

2. Sax, G., Cormack, T. R. (1966) "The effects of various forms of item arrangements on test performance," Journal of Educational Measurement, 22, 371-376.

3. Leary, L. F., Dorans, N. J. (1985) "Implications for altering the context in which test items appear: A historical perspective on an immediate concern," Review of Educational Research, 55 (3), 387-413.

4. Hestenes, D., Wells, M., Swackhamer, G. (1992) "Force Concept Inventory," The Physics Teacher, 30, 141-157.

5. Rebello, N. S., Zollman, D. A. (2002) "The effect of distracters on student performance on the Force Concept Inventory," PER: A Supplement to the American Journal of Physics, accepted for publication.

6. Howell, D. C. (1999) "Fundamental Statistics for the Behavioral Sciences," $4^{\text {th }}$ Ed., Duxbury Press, Pacific Grove, CA, pg. 95. 